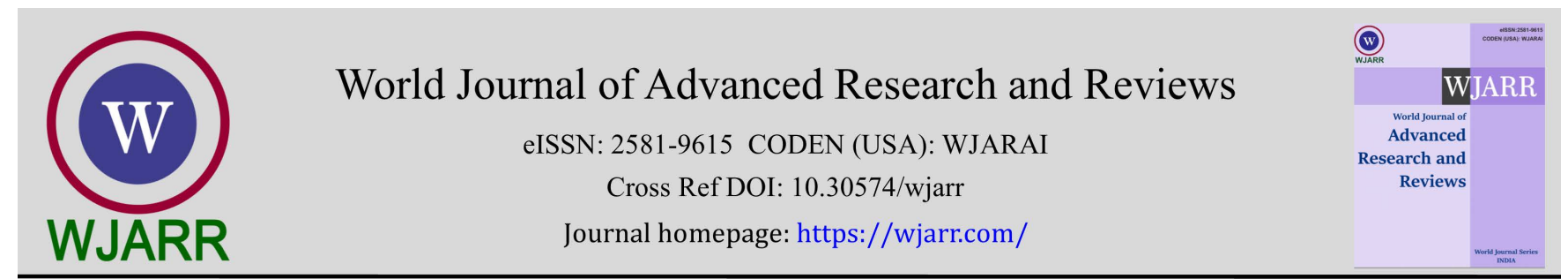

(REVIEW ARTICLE)

\title{
A review study of Cerebral palsy as a motor disability syndrome in children
}

\author{
Lekpa K David ${ }^{1}$ and Willy B Vidona ${ }^{2, *}$ \\ ${ }^{1}$ Department of Anatomy, University of Port Harcourt, Rivers State, Nigeria. \\ 2 Department of Anatomy, Edo State University Uzairue, Edo State, Nigeria.
}

World Journal of Advanced Research and Reviews, 2021, 12(01), 112-116

Publication history: Received on 30 August 2021; revised on 01 October 2021; accepted on 03 October 2021

Article DOI: https://doi.org/10.30574/wjarr.2021.12.1.0418

\begin{abstract}
Cerebral palsy is non-progressive motor disability syndrome largely attributed to abnormal development or damage in one or more parts of the brain especially the cerebellum and frontal lobe of the cerebrum that control muscle tone and motor activity and causing variable mental, motor and behavioral dilemmas. The study aims to review the information on the varied causes, types and clinical manifestation, diagnostic examination and management in cerebral palsy children. Prominent clinical presentation in this study was delayed developmental milestones such as delayed and coordinated movement of limbs, speaking delay and ptosis. Causes were noted to be pronounced in children born with low weight, preterm babies, trauma, delay among other complications during labor.
\end{abstract}

Keywords: Cerebral children; Cerebral palsy; Clinical manifestations; Delayed milestone; Imaging tools; Motor disability

\section{Introduction}

Cerebral palsy as a term is used to describe group of movement disorders as it affects body movements and coordination in early childhood [1], [2]. Cerebral palsy is, in fact a common disorder of children with clinical presentation of a wide variety of cerebral cortical or sub-cortical insults occurring during the first year of life, either pre, during and after birth [3]. The brain primarily the central nervous system is vulnerable and easily harmed during an early period of development by complications of prematurity such as and periventricular leukomalacia and intraventricular or intracranial hemorrhage due to fetal stroke from blood clots in the placenta that block blood flow [4].

Damage to one or more specific areas of the brain such as the cerebellum that control muscle tone and frontal lobe of the cerebrum that control motor activity seems to be the attributed location by correlations with the clinical manifestation.

\section{Causes of cerebral palsy}

Causes of cerebral palsy are varied from congenital in nature to well established causative indices in cases of stress or complications observed during labor that might have caused asphyxia which according to Pharaoh, [5] is estimated in about 5\%-10\% of children' birth as well as developmental infections [6] or even maternal low blood pressure, rupture of the uterus, detachment of the placenta, or problems involving the umbilical cord [7]. Although newborn's blood is equipped to compensate for short-term low levels of oxygen, if the supply of oxygen is reduced for lengthy periods, which is usually the case in scenario of insufficient, competent and responsive health practitioner within the environment thus resulting to an infant developing brain damage of hypoxic-ischemic encephalopathy type, which destroys tissue in the cerebral motor cortex of the brain.

\footnotetext{
${ }^{*}$ Corresponding author: Willy B Vidona; E mail: Wills_bills@yahoo.com

Department of Anatomy, Edo State University Uzairue, Edo State, Nigeria.

Copyright (c) 2021 Author(s) retain the copyright of this article. This article is published under the terms of the Creative Commons Attribution Liscense 4.0.
} 
According to a report by William and Carey [8] between $40 \%$ and $50 \%$ of all children who develop cerebral palsy were born prematurely, of which most of these cases (75-90\%) are believed due to issues that occur around the time of birth, often just after birth [9] (John, 2013). Multiple-birth infants are also more likely a pre-disposing factor than single-birth infants to having cerebral palsy [10].

Other causes could be as a result of interruption of the normal process of brain growth during fetal development likely due to gene mutation that interfere with the transmission of brain signals which is particularly vulnerable during the first 20 weeks of development.

It is very common on observation that most children who are born with cerebral palsy have more than one risk factor associated with it [11].

\section{Clinical manifestations of cerebral palsy}

Signs of cerebral palsy are usually not noticeable in early infancy but become more obvious as the child's nervous system matures and the problems and disabilities could range from very mild to very severe depending on the severity of the brain damage. They may be very subtle, noticeable only to medical professionals, or may be obvious to the parents and other caregivers. Signs and symptoms vary among people and over time with the obvious as follows:

i. Delayed milestones such as controlling head, rolling over, reaching with one hand, sitting without support, crawling, or walking usually accompanied with abnormal muscle tone which may be very stiff (spastic) even at joints as well or unusually relaxed and floppy leading to limbs being held in coordinating positions by muscle exertion of differing tone or strength.

ii. Abnormal movements that may be unusually jerky or slow and writhing that may appear uncontrolled or without purpose usually from skeletal deformities that may manifest in shortened limbs on the affected side and if not corrected by surgery or a device can lead to tilting of the pelvic bones and scoliosis.

iii. Seizures noticeable in about one third of people with cerebral palsy have seizures which may appear early in life or years later with the physical signs that may be partly masked by the abnormal movements of a person with cerebral palsy and sometimes epilepsy [12].

iv. Vision problems noticeable in three quarters of people with cerebral palsy have strabismus or ptosis due to weakness of the muscles that control eye movement which may result to nearsightedness if severe over time and sometimes weak eyelid muscle leading to dropping of upper eyelid.

v. Speech problems is seen in some individuals with cerebral palsy with the inability to control tongue muscles and thus cannot speak normally quite early which often times is accompanied by total/partial hearing loss in severe cases [13].

vi. Swallowing problems/Drooling shown by inability to control muscles involved in sucking, eating, drinking, and controlling their saliva [13], [14]. This is occasionally accompanied by dental problems with noticeable cavities than usual that can results from both defects in tooth enamel and difficulties brushing the teeth.

vii. Bowel and/or bladder control problems with an average of 55.5\% of people with cerebral palsy experiencing lower urinary tract symptoms, more commonly excessive storage issues than voiding issues [15] due to lack of muscle control.

viii. Children born with cerebral palsy are more likely to have some learning disabilities, which may be unrelated to their intelligent quotient (IQ) [16] with different patients likely to manifest different degrees of intellectual ability [17] that varies from genius to intellectual impairment [18].

In fact children with cerebral palsy suffer from multiple problems and potential disabilities that require the provision of family-centered services that make a difference in the lives of these children and their [19].

\section{Diagnostic examinations of cerebral palsy}

The diagnosis of cerebral palsy has historically rested on the child's history and physical examination. A general movement's assessment which involves measuring movements that occur spontaneously among those less than four months of age, appears most accurate [20] usually child's milestone movement, although children who are more severely affected are more likely to be noticed and diagnosed earlier [21]. Symptoms and diagnosis typically occur by the age of two, [22] although patients with milder forms of the condition may be over the age of 5, if not in adulthood, when finally diagnosed. Early diagnosis and intervention are seen as being a key part of managing cerebral palsy ([23]. 
Early detection and diagnosis is critical since it allows medical practitioners to begin treatment when the disorder is in its initial stages. Neuroimaging with Computed Tomography (CT) or Magnetic Resonance Imaging (MRI) is warranted when the cause of a person's cerebral palsy has not been established, however once a person is diagnosed with cerebral palsy, further diagnostic tests are optional. An MRI is preferred over CT, due to diagnostic yield and safety. When abnormal, the neuroimaging study can suggest the timing of the initial damage [24].

The challenges of management of cerebral palsy begin from diagnosis to the provision of care long after diagnosis. The ability to provide a diagnosis for the disorder early in the child's life may determine the success of treatment process.

\section{Management of cerebral palsy}

Management of cerebral palsy is observed to be primarily for the purpose of increasing functionality, improving capabilities, sustaining health in terms of locomotion, cognitive development, social interaction, and independence as rightly pointed by Taylor [25]. The best clinical outcomes comes from early intensive management involving multidisciplinary team approach of physical therapists, orthopedic surgeons with focus on total patient development, not just on improvement of a single symptom; as this provides the best model for medical care of patients across their lifespan to manage various associated and secondary conditions as well as address support system and psychosocial needs [26].

It is important that children with cerebral palsy be screened for mental retardation, ophthalmologic and hearing impairment, speech and language disorders and nutrition and growth should be monitored [27].

Equally care programs is seen to encompass physical and behavioural therapy, pharmacologic and surgical treatments, mechanical aids, and management of associated medical conditions according to Tilton [28]. While occupational and behavioural therapy seeks to enhance patient-caregiver interactions, Physical therapy is more of a post-operative management procedure.

\section{Conclusion}

Cerebral palsy is a chronic motor disorder that is said to affect the parts of the brain, with unknown cause in most cases although birth trauma and prematurity remains the commonest risk factor. Children with cerebral palsy suffer from multiple problems and potential disabilities among which are abnormal muscle tone, delayed motor development, and delayed speech all classified as delayed developmental milestones. Screening for these conditions should be part of the initial assessment for proper attention and care which is more effective on an individualized treatment plan that provides a combination of interventions and that requires the provision of a number of family centered services so as to improve the quality of life through a coordinate a complex care system that maximize the capabilities and benefits of the child.

\section{Recommendation}

It is recommended that computed tomography of the brain (when MRI was not available) be the least to deployed in suspected cases of cerebral palsy to establish any brain abnormality. Further metabolic and genetic tests are recommended to exclude underlying genetic or metabolic etiology especially those with malformations. Those with focal vascular insult, coagulation studies are recommended to exclude coagulopathy.

Cerebral palsy is non- curable in the accepted sense although several measures such as proper education, therapy and applied technology are being used to help persons who are suffering from this disorder and provide them productive lives. In order to approach cerebral palsy systematically, the medical practitioners and physical therapists need to recognize neuromotor deficits, diagnose and implement a methodical treatment plan.

\section{Compliance with ethical standards}

\section{Acknowledgments}

The authors are immensely grateful to Mrs. Charity Willy-Vidona and Mrs. Courage Lekpa David for the social support as well as type setting and proof reading of manuscript. 


\section{Disclosure of conflict of interest}

The authors, Dr. Lekpa K. David and Dr. Willy B. Vidona hereby declare that there is no conflict of interests.

\section{Statement of ethical approval}

The authors declared that this study has received no approval as no human subjects were applied.

\section{References}

[1] Haak P, Lenski M, Hidecker MJC, Li M, Paneth N. Cerebral palsy and aging. Developmental Medicine \& Child Neurology. 2009; 51: 16-23.

[2] Amrita K, Yadav S. Cerebral Palsy: a Mini Review, Department Of Pharmacy, Banasthali University International, Journal of Therapeutic Applications. 2012; 3: 15 - 24.

[3] Beukelman RD, Mirenda P. Augmentative and Alternative Communication: Management of severe communication disorders in children and adults (2nd ed.). Baltimore: Paul H Brookes Publishing Co. 1999; 246249.

[4] Tanaka A, Araki JI, Tasaki A, Miyamoto KC. Improvement of hypertonus after treatment for sleep disturbances in three patients with severe brain damage. Brain Development. 1997; 19: 240-244.

[5] Pharoah PO. Causal hypothesis for some congenital anomalies. Twin Res Hum Genet. 2005; 8(6): 543-550.

[6] Nelson KB, Blair E. Prenatal Factors in Singletons with Cerebral Palsy Born at or near Term. The New England Journal of Medicine. 2015; 373(10): 946-53.

[7] Lindquist BH. Bruxism in children with brain damage. Acta Odontal Scandinavian. 1999; 32(5): 313-19.

[8] William B, Carey D. Developmental-behavioral pediatrics (4th ed.). Philadelphia, PA: Saunders/Elsevier. 2009; 264.

[9] John Y. Epidemiology and Disease Prevention: A Global Approach (02 ed.). Oxford University Press. 2013; 190.

[10] Saunders NR, Hellmann J, Farine D. Cerebral palsy and assisted conception. Journal of Obstetrics and Gynaecology Canada. 2011; 33(10): 1038-43.

[11] Eunson P. Aetiology and epidemiology of cerebral palsy. Paediatrics and Child Health. 2016; 26(9): 367-372.

[12] Rosenbaum P, Paneth N, Leviton A. A report: the definition and classification of cerebral palsy. Dev Med Child Neurol Suppl. 2007; 109: 8-14.

[13] Oskoui M, Coutinho F, Dykeman J, Jetté N, Pringsheim T. Cerebral Palsy: Hope Through Research. National Institute of Neurological Disorders and Stroke. 2013; 55(6): 509-19.

[14] Walshe M, Smith M, Pennington L. Interventions for drooling in children with cerebral palsy. Walshe, Margaret (ed.). The Cochrane Database of Systematic Reviews. 2012; 11(8): 6-24.

[15] Samijn B, Van Laecke E, Renson C, Hoebeke P, Plasschaert F, Vande Walle J, Van den Broeck C. Lower urinary tract symptoms and urodynamic findings in children and adults with cerebral palsy: A systematic review. 2016; 36(3): 541-549.

[16] Marwa O. Role of piracetam in treatment of cerebral palsy disease. Journal of Behavioral Health. 2012; 1(1): 5358.

[17] Eric C, Ann R. Management and Outcomes of Very Low Birth Weight. The New England Journal of Medicine. 2008; 358(21): 1700-1711.

[18] Neil M. Neurologic and Developmental Disability at Six Years of Age after Extremely Preterm Birth. The New England Journal of Medicine. 2005; 352(14): 9-19.

[19] King S, Teplicky R, King G, Rosenbaum P. Family centered service for children with cerebral palsy and the families: a review of the literature. Semin Pediatr Neurol. 2004; 11(1): 78-86.

[20] McIntyre S, Morgan C, Walker K, Novak I. Cerebral palsy-don't delay. Developmental Disabilities Research Reviews. 2011; 17(2): 114-29. 
[21] Bosanquet M, Copeland L, Ware R, Boyd R. A systematic review of tests to predict cerebral palsy in young children. Developmental Medicine \& Child Neurology. 2013; 55(5): 418-26.

[22] Lungu C, Hirtz D, Damiano D, Gross P, Mink JW. Report of a workshop on research gaps in the treatment of cerebral palsy. Neurology. 2016; 87(12): 1293-1298.

[23] Graham D, Paget SP, Wimalasundera N. Current thinking in the health care management of children with cerebral palsy. Medical Journal of Australia. 2019; 210(3): 129-135.

[24] Kolawole TM, Patel PJ, Mahdi AH. Computed tomographic (CT) scans in cerebral palsy (CP). Pediatric Radiology. 1999; 20(1-2): 23-27.

[25] Taylor F. National Institute of Neurological Disorders and Stroke (U.S.) Office of Science and Health Reports. Cerebral palsy: Hope through research. Bethesda, Md. The Institute. 2001.

[26] Johnston M, Hagberg H. Sex and the pathogenesis of cerebral palsy. Dev Med Child Neurol. 2007; 49: 74-8.

[27] Ashwal S, Russian BS, Blasco PA, Miller G, Sandler A, Shivell M, Stevenson R. Practice Parameter: Diagnostic assessment of the child with cerebral palsy. Neurology. 2004; 62(6): 851-863.

[28] Tilton AH. Management of spasticity in children with cerebral palsy. Semin PediatrNeurol. 2004; 11(1): 58- 65. 